\title{
Awake craniotomy anesthetic management using dexmedetomidine, propofol, and remifentanil
}

This article was published in the following Dove Press journal:

Drug Design, Development and Therapy

3 March 2017

Number of times this article has been viewed

\author{
Andrea Prontera' \\ Stefano Baroni ${ }^{2}$ \\ Andrea Marudi ${ }^{2}$ \\ Franco Valzania ${ }^{3}$ \\ Alberto Feletti' \\ Francesca Benuzzi ${ }^{4}$ \\ Elisabetta Bertellini ${ }^{2}$ \\ Giacomo Pavesi' \\ 'Department of Neurosurgery, Nuovo \\ Ospedale Civile SAgostino-Estense, \\ ${ }^{2}$ Department of Anesthesiology, \\ Nuovo Ospedale Civile SAgostino- \\ Estense, ${ }^{3}$ Department of \\ Neurology, Nuovo Ospedale Civile \\ S Agostino-Estense, ${ }^{4}$ Department of \\ Neuroscience, University of Modena \\ and Reggio Emilia, Modena, Italy
}

\begin{abstract}
Introduction: Awake craniotomy allows continuous monitoring of patients' neurological functions during open surgery. Anesthesiologists have to sedate patients in a way so that they are compliant throughout the whole surgical procedure, nevertheless maintaining adequate analgesia and anxiolysis. Currently, the use of $\alpha 2$-receptor agonist dexmedetomidine as the primary hypnotic-sedative medication is increasing.

Methods: Nine patients undergoing awake craniotomy were treated with refined monitored anesthesia care (MAC) protocol consisting of a combination of local anesthesia without scalp block, low-dose infusion of dexmedetomidine, propofol, and remifentanil, without the need of airways management.
\end{abstract}

Results: The anesthetic protocol applied in our study has the advantage of decreasing the dose of each drug and thus reducing the occurrence of side effects. All patients had smooth and rapid awakenings. The brain remained relaxed during the entire procedure.

Conclusion: In our experience, this protocol is safe and effective during awake brain surgery. Nevertheless, prospective randomized trials are necessary to confirm the optimal anesthetic technique to be used.

Keywords: dexmedetomidine, awake surgery, anesthesia

\section{Abbreviations}

bpm, beats per minute; DEX, dexmedetomidine; EcoG, electrocorticography; HR, heart rate; LMA, laryngeal mask airway; MAC, monitored anesthesia care; MAP, mean arterial pressure; MEPs, motor evoked potentials; PRO, propofol; REM, remifentanil; SEPs, somatosensory evoked potentials.

\section{Introduction}

Awake craniotomy enables neurosurgeons to continuously monitor the patient's neurological functions during open surgery.

Anesthesiologists are required to maintain the patient's cooperation along with adequate sedation, analgesia, and anxiolysis throughout the whole procedure. Although awake craniotomy procedures have become common in neurosurgical practice, there is still no consensus on the optimal pharmacological regimen during the intervention.

The use of $\alpha 2$-receptor agonist DEX as primary hypnotic-sedative medication is common nowadays, especially in intensive care unit settings, because it provides effective and safe conscious sedation.

DEX rapidly acts on the pontine locus coeruleus, providing subcortical sedation characterized by a comfortable and quick arousal, with minimal effect on the respiratory drive. Bradycardia and moderate hypotension are the most common hemodynamic effects.

\footnotetext{
Correspondence: Alberto Feletti Department of Neurosurgery, Nuovo Ospedale Civile S Agostino-Estense Modena Hospital, via Pietro Giardini, I355, Modena 4| | 26, Italy

Tel +39059396 I47|

Fax +390593962408

Email alberto.feletti@gmail.com
} 
We describe the anesthetic management of patients who underwent awake craniotomies at our institution (NOCSAE Modena Hospital, Modena, Italy). A combination of DEX, PRO, and REM was used without any need for airway instrumentation.

\section{Methods}

\section{Population}

We retrospectively reviewed all awake craniotomies performed with MAC protocol at our institution from May 2013 to December 2014. We identified 9 patients ( 5 males and 4 females) meeting the inclusion criteria. They underwent craniotomy to remove 7 gliomas, 1 meningioma, and 1 cavernoma. Unfavorable anatomical features such as short neck or overweight were not considered exclusion criteria. Data regarding anesthetic technique, vital parameters, and patients' outcome were collected.

The most involved locations were the middle frontal gyrus, the middle temporal gyrus, the precentral gyrus, the inferior frontal gyrus, the angular gyrus, and the superior temporal gyrus.

Patients' demographics and pathological characteristics are shown in Table 1.

A neuropsychologist instructed the patients to perform the neurological examination during surgery.

The official institutional board (Modena Local Health Authority - Internal Review Board) approved the study. Patient consent was not required by the institutional board to review medical records.

\section{Anesthetic and surgical procedures}

All patients underwent awake craniotomy using the MAC protocol.

Ranitidine $50 \mathrm{mg}$ and metoclopramide $10 \mathrm{mg}$ were administered $2 \mathrm{~h}$ before induction of anesthesia to prevent nausea and vomiting.

After electrocardiography, pulse oximetry, noninvasive blood pressure monitoring, and double peripheral venous cannulation, we started the simultaneous infusion of DEX, PRO, and REM.

Radial artery-line, Foley catheter, and an additional peripheral intravenous catheter were placed after induction of anesthesia.

Initial DEX dose of $0.7 \mu \mathrm{g} / \mathrm{kg} / \mathrm{h}$ was followed by $0.2-1.4 \mu \mathrm{g} / \mathrm{kg} / \mathrm{h}$ maintenance infusion. No loading dose was used, and infusion rate was progressively increased until adequate sedation was obtained. We used Ramsay sedation score to assess the level of sedation every 90 s. A Ramsey sedation score between 4 and 6 was considered adequate. It was reached by slowly increasing the DEX infusion to $0.2 \mu \mathrm{g} / \mathrm{kg}$, unless the HR decreased to $50 \mathrm{bpm}$ or the MAP decreased to $60 \mathrm{mmHg}$. In case of persistently inadequate sedation despite these adjustments, we increased the concentration of the PRO infusion.

All patients had spontaneous ventilation with supplemental oxygen $(2 \mathrm{~L} / \mathrm{min}$ ) through a capnomask (Mediplus, Bucks, UK). No laryngeal mask or other devices were used.

A 3-pin head-holder was placed on the patient under local anesthesia with subcutaneous injection of bupivacaine with epinephrine $(1: 200,000)$ and ropivacaine $7.5 \mathrm{mg} / \mathrm{mL}$, without nerve block.

Comfortable positioning of the patient was achieved, keeping the head slightly elevated in order not to compress jugular venous flow. Such positioning prevents airway occlusion during the asleep period, especially in patients with anatomically unfavorable neck.

Patients underwent EcoG using a 32-channel Nicolet alliance portable unit (Nicolet BMSI, Inc, Madison, WI, USA). Standard MEPs and SEPs were recorded.

Surgical incision was planned using neuronavigation in order to expose both the tumor and the surrounding cortical eloquent areas. A solution of bupivacaine with epinephrine $(1: 200,000)$ and ropivacaine $7.5 \mathrm{mg} / \mathrm{mL}$ was used for local anesthesia.

Draping was adjusted to keep eyes and airways easily accessible.

Table I Patient demographics and clinicopathological characteristics

\begin{tabular}{|c|c|c|c|c|c|c|}
\hline Number & Age, years & Sex & Previous history & Pathology & Grade & Location (gyrus) \\
\hline I & 48 & $M$ & Glaucoma & Glioma & IV & Posterior T2-T3 \\
\hline 2 & 57 & $\mathrm{~F}$ & Hypertension & Meningioma & I & Precentral-F3 \\
\hline 3 & 56 & $\mathrm{~F}$ & $\begin{array}{l}\text { Hypertension, obesity, } \\
\text { diabetes mellitus type } 2\end{array}$ & Glioma & III & Posterior FI \\
\hline 4 & 62 & $\mathrm{~F}$ & Dyslipidemia & Glioma & II & $\mathrm{F} 2$ \\
\hline 5 & 30 & $M$ & None & Glioma & II & Precentral \\
\hline 6 & 31 & $\mathrm{~F}$ & None & Cavernoma & & $\mathrm{F} 2$ \\
\hline 7 & 41 & $M$ & None & Glioma & II & T2-angular \\
\hline
\end{tabular}

Abbreviations: $F$, female; $M$, male. 
The patient was maintained asleep during scalp incision, bone flap removal, and dural opening. Dura mater was soaked with lidocaine $10 \%$ before incision.

After brain exposure, all drugs were stopped, and the patient progressively awakened. The surgeon gave ongoing feedback to the anesthesiologist regarding the brain appearance, in order to maintain the brain relaxed.

Cortical mapping was achieved using Ojemann cortical stimulator (Radionics Corp, Burlington, MA, USA) with a monopolar probe, delivering stimuli with a single $1 \mathrm{~ms}$ pulse with a $60 \mathrm{~Hz}$ frequency. Skill tests were performed during surgical resection.

Analgesics (acetaminophen $1 \mathrm{~g}$ ) were added when needed.

When resection adjacent to functionally relevant areas was completed, anesthesia was reinduced with DEX, PRO, and REM infusions titrated to patient sedation level.

Patients spontaneously breathed throughout the whole procedure without the aid of a laryngeal mask.

After wound closure, all anesthetics were stopped, allowing the patient to awaken from anesthesia in the operative theater. All patients were admitted to the subintensive care unit.

\section{Results}

Table 2 summarizes the drug infusion doses, HR, arterial blood pressure, MAP, awakening periods, and length of anesthesia.

From induction to awakening, DEX, PRO, and REM infusion doses ranged between 0.28 and $0.90 \mu \mathrm{g} / \mathrm{kg} / \mathrm{h}, 0.51$ and $1.26 \mathrm{mg} / \mathrm{kg} / \mathrm{h}$, and 0.02 and $0.05 \mu \mathrm{g} / \mathrm{kg} / \mathrm{min}$, respectively.

We observed HR and MAP decrease as expected; however, their values were never less than $50 \mathrm{bpm}$ and $60 \mathrm{mmHg}$, respectively.

EcoG confirmed appropriate patient sedation.

After stopping drug infusion, the patients were able to collaborate for an average period of $10 \mathrm{~min}$. The awakening was always smooth, resembling natural awakening. Moreover, as we avoided the use of a laryngeal mask and other airway devices, we did not observe any coughing.

The awakening period lasted on average 77 min (range: 40-120 $\mathrm{min}$ ). Three patients required additional analgesia (acetaminophen $1 \mathrm{~g}$ ).

Drugs infusion doses during the final part of the operation ranged between 0.23 and $0.91 \mu \mathrm{g} / \mathrm{kg} / \mathrm{h}$ for DEX, 0.2 and $1.45 \mathrm{mg} / \mathrm{kg} / \mathrm{h}$ for PRO, and 0.04 and $0.08 \mu \mathrm{g} / \mathrm{kg} / \mathrm{min}$ for REM. Similar to the previous asleep period, we did not observe critical levels of HR and MAP.

After surgery, all patients reached an Aldrete score of 10 after a mean period of 11 min (range: 4-22 min).
No significant respiratory depression was noticed. Intraoperative arterial blood gas analysis confirmed normoxia and normocapnia.

\section{Discussion}

In the past few years, awake craniotomy has allowed wider and safer surgical resections of lesions located in the vicinity of functionally relevant areas.

The possibility to easily wake the patients up and neurologically monitor them during surgery is the main advantage of this procedure.

However, there is still no consensus or any established protocol for the best anesthetic management of such perioperative sedation. ${ }^{1}$

Recently, DEX, a selective alpha-2 receptor agonist, has been proposed as the primary sedative-hypnotic drug, as it does not depress respiratory drive and it does not interfere with electrophysiological testing. Its sedative effects are mediated through decreased firing of the locus coeruleus, the main noradrenergic nucleus located on the floor of the fourth ventricle, and subsequent decrease in noradrenaline release in the sympathetic nerves. Such characteristics encourage the use of DEX during awake neurosurgical procedures.

However, DEX has well-known adverse effects, such as bradycardia and hypotension. These cardiovascular effects are dose dependent: with lower infusion rates, the central effect predominates, leading to a decrease in HR and blood pressure. With higher doses, peripheral vasoconstriction prevails, leading to an increase in systemic vascular resistance and blood pressure, while the bradycardic effect is further emphasized. Cardiac arrest and respiratory depression have also been reported. ${ }^{2,3}$ Several studies ${ }^{4,5}$ have extensively shown the role of DEX during functional neurosurgery. We reviewed the pertinent literature about the use of DEX during awake surgery in functionally relevant brain areas.

de Almeida et $\mathrm{al}^{6}$ operated on 3 epileptic patients with cavernous angiomas in the speech area using DEX as the primary hypnotic drug, without the need to protect airway patency. They administered boluses of fentanyl, midazolam, PRO, and droperidol during the procedure when judged necessary. In their series, they did not report either significant hemodynamic instability or respiratory depression.

Shen et $\mathrm{al}^{7}$ prospectively compared DEX and PRO as primary hypnotic drugs during awake craniotomy, performed with asleep-awake-asleep protocol. They concluded that both DEX and PRO can be effectively and safely administered for conscious sedation. However, they found that DEX has a shorter arousal time. 


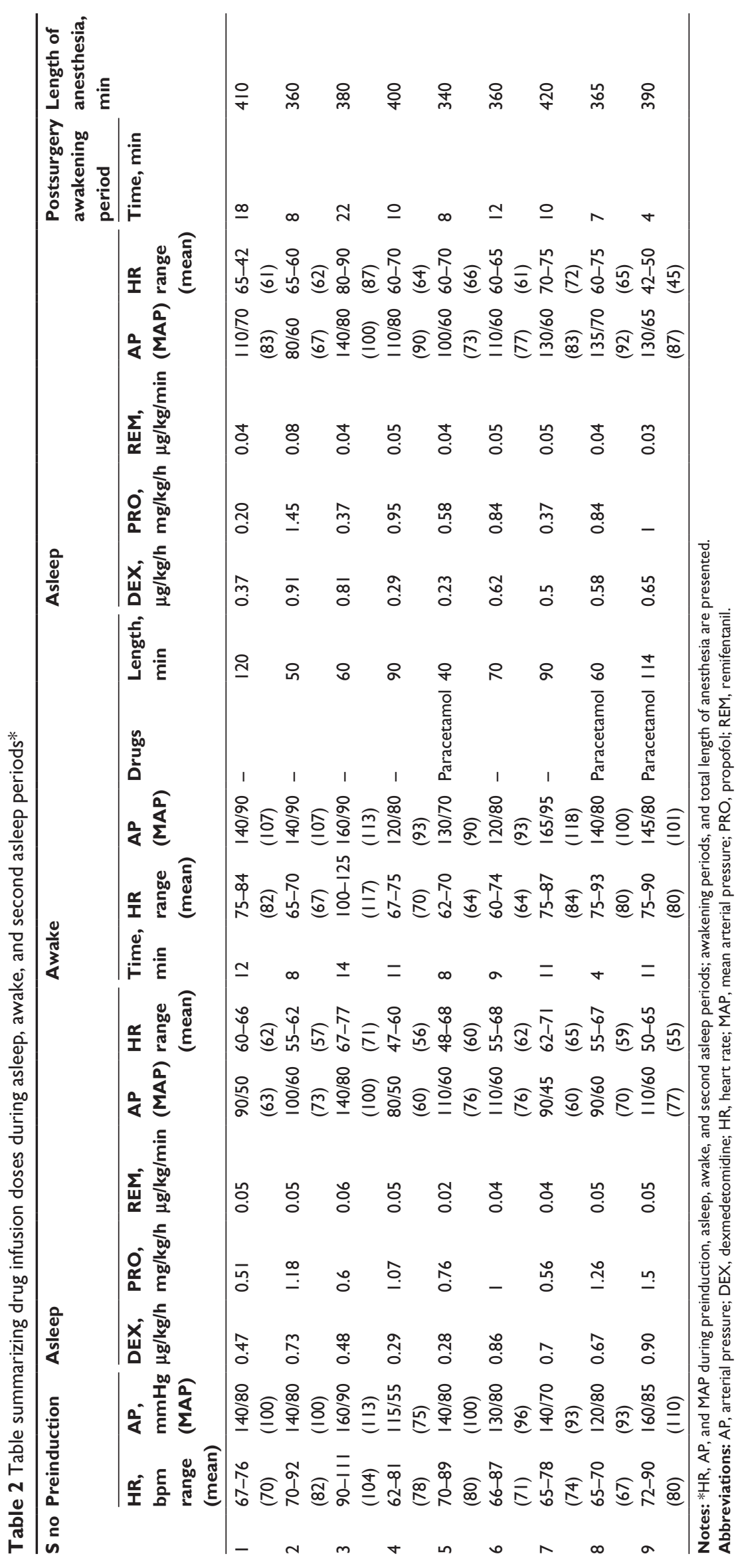


Mohd Nazaruddin et $\mathrm{al}^{8}$ successfully treated 4 patients with a combination of DEX and REM intravenous infusions and scalp block with the MAC protocol. They delivered oxygen to patients only through nasal prongs. Only 1 patient required supplementary infusion of PRO to achieve adequate sedation.

Kallapur and Bhosale ${ }^{9}$ reported a case of tumor removal with awake craniotomy, using scalp block without 3-pin holder, with simultaneous infusion of DEX and PRO and oxygen delivery through a simple mask. They concluded that DEX is a useful adjuvant during awake craniotomy for tumor resection.

Chung et $\mathrm{al}^{10}$ performed 6 different anesthetic managements during awake tumor removal in 6 different patients. They simultaneously infused DEX, PRO, and REM in 2 patients. In 1 case, without airway protection, they did not obtain adequate sedation despite a high dose of sedatives. In the second patient, they reported good sedation without perioperative events, although with the aid of LMA.

Mack et $\mathrm{al}^{11}$ presented a retrospective review of 10 patients who received DEX for awake craniotomy. Five of them were managed with MAC protocol. They concluded that DEX appears to be a useful sedative for awake craniotomy.

In our study, we applied a refined MAC protocol to perform awake craniotomy. We obtained adequate sedation and analgesia with the simultaneous infusion of DEX, PRO, and REM without any loading dose. Using a combination of drugs rather than a single drug presents several advantages. First, this regimen allows the use of lower PRO doses, thus reducing the risk of respiratory depression. Second, DEX doses can also be reduced, thus decreasing the risk of cardiovascular side effects. Third, this analgosedative regimen allows spontaneous airway patency without the need for tracheal intubation, LMA, or nasal airway, therefore reducing the risk of cough during the awake period. All patients were able to breathe spontaneously.

We did not perform scalp block, despite the use of a 3-pin head holder. We provided proper local anesthesia by injecting only the 3 sites around the pins and the skin incision. Actually, avoiding scalp block can reduce the risk of eventual transient bacteremia due to multiple needle-stick injuries, at the same time ensuring a comfortable surgical experience to the patient.

After stopping the infusions, all patients experienced smooth and rapid awakening. The brain remained relaxed throughout the entire procedure. Similarly, we also observed stability of hemodynamic parameters during the period of neurological monitoring.
Only 3 patients complained of mild pain, successfully treated with acetaminophen. Two patients experienced dry mouth.

We also observed that a small increase of PRO dose during simultaneous infusion of DEX leads to a higher-thanexpected increase of respiratory frequency.

The main limitations of our study are the limited number of included patients, as well as the retrospective design of the study.

\section{Conclusion}

Awake craniotomy entails specific anesthetic management. DEX is a unique sedative agent that does not directly cause respiratory depression. At the moment, the optimal dose regimen of DEX during awake craniotomy is unknown. Within the limits of a retrospective study conducted on a limited number of patients, we have refined a MAC protocol combining local anesthesia without scalp block and low-dose infusion of DEX, PRO, and REM, with no need for airway support. This protocol proved safe and effective for this surgery. Nevertheless, prospective randomized trials are necessary to confirm the optimal anesthetic technique in this setting.

\section{Acknowledgment}

The authors thank Dr Antonella Fontana for editing the manuscript.

\section{Disclosure}

The authors report no conflicts of interest in this work.

\section{References}

1. Piccioni F, Fanzio M. Management of anesthesia in awake craniotomy Minerva Anestesiol. 2008;74(7-8):393-408.

2. Bharati S, Pal A, Biswas C, Biswas R. Incidence of cardiac arrest increases with the indiscriminate use of dexmedetomidine: a case series and review of published case reports. Acta Anaesthesiol Taiwan. 2011;49(4) 165-167.

3. Ha SH, Park I-H, Lee M-H, Shi S-K, Min KT. Use of dexmedetomidine for awake craniotomy. Korean J Anesthesiol. 2011;61(4):346-347.

4. Erickson KM, Cole DJ. Anesthetic considerations for awake craniotomy for epilepsy and functional neurosurgery. Anesthesiol Clin. 2012;30(2): 241-268.

5. Rozet I, Muangman S, Vavilala MS, et al. Clinical experience with dexmedetomidine for implantation of deep brain stimulators in Parkinson's disease. Anesth Analg. 2006;103(5):1224-1228.

6. de Almeida AN, Tavares C, Tibano A, Sasaki S, Murata KN, Marino R Jr. Dexmedetomidine for awake craniotomy without laryngeal mask. Arq Neuropsiquiatr. 2005;63(3B):748-750.

7. Shen S, Zheng J, Zhang J, et al. Comparison of dexmedetomidine and propofol for conscious sedation in awake craniotomy: a prospective, double-blind, randomized, and controlled clinical trial. Ann Pharmacother. 2013;47(11):1391-1399. 
8. Mohd Nazaruddin WH, Mohd Fahmi L, Laila AM, Zamzuri I, Abdul Rahman IZ, Hardy MZ. Awake craniotomy: a case series of anaesthetic management using a combination of scalp block, dexmedetomidine and remifentanil in Hospital Universiti Sains Malaysia. Med J Malaysia. 2013;68(1):64-66.

9. Kallapur BG, Bhosale R. Use of dexmedetomidine infusion in anaesthesia for awake craniotomy. Indian J Anaesth. 2012;56(4):413-415.
10. Chung YH, Park S, Kim WH, Chung IK, Lee JJ. Anesthetic management of awake craniotomy with laryngeal mask airway and dexmedetomidine in risky patients. Korean J Anesthesiol. 2012;63(6):573-575.

11. Mack PF, Perrine K, Kobylarz E, Schwartz TH, Lien CA. Dexmedetomidine and neurocognitive testing in awake craniotomy. J Neurosurg Anesthesiol. 2004;16(1):20-25.

\section{Publish your work in this journal}

Drug Design, Development and Therapy is an international, peerreviewed open-access journal that spans the spectrum of drug design and development through to clinical applications. Clinical outcomes, patient safety, and programs for the development and effective, safe, and sustained use of medicines are the features of the journal, which has also been accepted for indexing on PubMed Central. The manuscript management system is completely online and includes a very quick and fair peer-review system, which is all easy to use. Visit http://www.dovepress.com/testimonials.php to read real quotes from published authors.

Submit your manuscript here: http://www.dovepress.com/drug-design-development-and-therapy-journal 